\title{
SOME ASPECTS OF THE PHYSIOLOGY OF CONTINENCE AND DEFAECATION*
}

\author{
BY \\ IAN P. TODD \\ From St. Bartholomew's Hospital, and St. Mark's Hospital, London
}

The physiology of defaecation and of faecal continence should be well understood by now, but in fact this can hardly be said to be true. A glance at a textbook readily confirms this. Most of us know more about the subject than is contained in the textbook, and some recent work confirms previous findings and throws much light on many difficult and little-understood problems.

I want to refer briefly to the use of multiple intraluminal pressure recorders, commonly called motility studies, and intrarectal balloons used in conjunction with electromyography of the muscles of the pelvic floor and the sphincters. Telemetering pills, sometimes referred to as 'gutniks', which register intraluminal pressure changes, confirm the findings by the simpler recording methods, but have not proved to be superior in the colon. A complete picture of function can only be obtained by combining motility, electromyographic and cineradiographic studies; this has not been done by us yet.

Distension of the normal rectum, at a critical volume, produces the call to stool and a moment later a beginning of inhibition of the external sphincter and pubo-rectalis muscles. Inhibition of these muscles, rather than relaxation of them, is probably the correct description, for the muscles show considerable resting activity at all other times (N. H. Porter, 1960; unpublished report).

The internal sphincter is not referred to in this paper, as it reflects the activity of the circular muscle of the intestine, of which it is a part. There is distal relaxation with proximal distension, and plastic adaptation occurs readily.

Porter has shown electromyographically that the external sphincter and the pubo-rectalis normally show heightened activity with effort, coughing and voluntary sphincteric contraction, but inhibition with defaecation and micturition. This is in keeping with the findings of previous investigators who did not appreciate, however, the continuous resting activity of the muscles. Other striated

\footnotetext{
* A paper read at a meeting of the British Association of Paediatric Surgeons in Stockholm, September 1961.
}

muscles in the body do not show resting activity with the electromyographic recording used.

A critical volume brings about the desire to stool and sphincteric inhibition. In many conditions this volume is abnormal, and although clinically one has been conscious of abnormal rectal volumes (as estimated by the size of stool passed), it has seldom been appreciated that this directly influences sphincteric inhibition and/or the call to stool.

If a large volume rectal balloon is used, which itself does not exert much pressure, and this is filled slowly until the call to stool is produced, some idea of this volume can be obtained. In the adult it is usually between $100-150 \mathrm{ml}$., though up to $200 \mathrm{ml}$. may be considered normal. Of course this figure can be obtained more simply if the average volume of stool passed is measured; this is, however, less accurate as, in many people, a conditioned reflex exists, bringing about evacuation with or without a sufficient call. This is particularly true of the 'once-a-day' man, in whom the call may be insignificant though urgency exists as the result of stimulation of the anal papillae.

If an unduly large volume is needed to bring about a call to stool, obviously infrequent bowel actions result, and thus a tendency to constipation. It seems that the call to stool and sphincteric inhibition usually go hand in hand. In one adult female $2,000 \mathrm{ml}$. failed to produce any sensation and clinically, though not proven electromyographically, there was no sphincteric relaxation. Similarly, a male child, aged 9 years, was thought to be a mild case of Hirschsprung's disease, because of prolonged periods of constipation without soiling. On examination, large faecal masses were apparent in the abdomen and rectum, and the anal sphincter was relaxed; after the rectum had been emptied, sphincter tone returned to normal. This suggests a large volume rectum and functional, not organic, disease.

In the child, the gauging of sphincter tone by means of a finger inserted into the anal canal is difficult, and it is better to part the buttocks forcibly 
and slowly, whilst watching the anus. If the sphincters are inhibited, the anus can be opened completely, if tonic, it cannot. In the adult, it is necessary to insert a finger and pull the anus back towards the coccyx; if the anus can be opened it is lax, either from inhibition or from some other cause.

An unduly small rectal volume producing a call and sphincteric inhibition may also be found. This may be accompanied by urgency. This occurs under several conditions. There may be a persistence of the neonatal habit of emptying the rectum each time something enters it, i.e. with each meal, when a gastro-intestino-colic reflex is set up. No conditioning has occurred in these cases to bring about a 'once-a-day' habit. Resections of part of the rectum by the abdomino-per-anal method may cause frequent small stools; here the sensory area is diminished in size to such an extent that stretching of the wall occurs rapidly. This is seen more often in adults than in children, whose rectums adapt better. Furthermore, the operation in the child is most commonly carried out for Hirschsprung's disease, when an aganglionic portion of bowel is left. Distension of the rectum or colon does not cause inhibition of an aganglionic segment. The results of resection in children and adults with Chagas' disease, due to the Trypanosoma cruzii, are initially good, but constipation tends to recur as further ganglia degenerate.

If frequent small stools accompanied by urgency are passed after a resection, complete sphincteric inhibition is being prevented by voluntary contraction. This is brought about by irritation of the secondary sensory area of anal papillae within the anal canal. This is the area which is normally only stimulated by liquid stools which can impinge upon the area more readily than solids. Voluntary contraction can only be maintained for something under a minute; thus Nature's call is urgent. If the subject lies down, the call fades, for the area is stimulated less.

Stool diameter is also important. A narrow stool is primarily a result of incomplete sphincteric inhibition and only rarely due to a narrowing of the lumen. A stool of some length, of normal diameter, means that inhibition is complete and functional rectal disease is most unlikely. A stool which is in the form of multiple spherical pellets suggests poor sensation and incomplete relaxation which leads to prolonged retention of faeces in the rectum and excessive water extraction. In the adult, this may occur in the colon when faeces are held up by narrowed irritable areas, such as exist with diverticulitis. Pellet stools may also suggest a local anal irritability and failure of normal inhibition, such as occurs with a fissure.

The narrow stool of normal shape is usually produced as the result of good bowel habit and insufficient faecal debris to cause complete sphincteric inhibition. As a result of an irritable bowel stimulating the anal area, either because the stool is liquid or because of colonic overactivity such as is seen in the spastic colon or mucous colitis, rectal distension and thus sphincteric inhibition does not occur, and so stool diameter is small.

This brings one to colonic activity. These records have been obtained from open-ended tubes or tiny balloons at normal body temperature and pressure, recorded photographically by means of a reflecting optic capsule. It has been shown that the colon is overactive in some cases of constipation, whilst the rectum is inactive. Electromyography sometimes shows delayed inhibition, the rectum requiring a large volume to produce any change in sphincter tone. Some of these cases show, in time, atonic megarectum and hypertrophic megacolon. Prostigmine stimulation tests have not helped these people. Occasionally, the use of an antispasmodic seems to do so, though it is difficult to say why this is so. It does not reduce colonic activity in the recording, but seems to bring about a heightened, regular and systematized contraction. Clearly, laxatives will not help in this type of case, for it is the rectum that is at fault, either primarily or secondarily. If secondary to prolonged overdistension, then re-eduction (keeping the rectum empty by means of enemas and suppositories) will in time bring success. If primarily, then surgical measures may be required.

Sometimes motility studies show excessive activity in rectum and colon, but electromyography may show delayed inhibition. This is a very unpleasant condition to treat, and laparotomy shows a hypertrophic colon and rectum, but no aganglionic segment. It may be due to a failure of the rectosphincteric inhibitory reflex, and leads to severe megacolon and megarectum.

Perhaps it should be mentioned that sometimes the sphincters are inhibited too easily or too completely, and resting activity returns more slowly than normal. These changes may be associated with rectal prolapse, in which sensation is either not developed or has been lost, according to the type and age group in which it occurs.

Perhaps too many aspects of the subject have been touched upon too superficially in this paper. It is not suggested that these investigations are needed in every case, though they are definitely helpful in difficult bowel problems. It is useful, however, to pay greater attention to the stool in 
cases showing an abnormal bowel habit. The volume of the stool passed suggests the degree of rectal distension that is required to produce sensation; the diameter of the stool reflects the degree of sphincteric inhibition; this, of course, depends also upon volume. The shape is important; length usually indicates good sphincteric inhibition; pellets indicate either irritability of the bowel or sphincter, or inadequate and too slow delivery of faecal remains in the rectum, allowing excessive water reabsorption before rectal distension has taken place. A terminal pointed end to the stool indicates a good return of reflex activity in the sphincter mechanism, and suggests a bowel that is well regulated. A stool that is not well cut off suggests a sluggish return of reflex activity or excessive inhibition of the sphincters, implying an excessive rectal distension and poor sensory response.

I am indebted to the Medical Research Council for the services of Dr. A. M. Connell for three years. He is now with their permanent research staff, and continues work on intestinal motility. Mr. Nigel Porter has carried out the electromyography, and to him I am most grateful. 